\title{
ACCIDENTES DEL PARTO
}

Doctor Carlos R. Silva-Mojica

Profesor Agregado de Clínica Obstétrica.

Este trabajo se basa en un estudio sobre 3.800 partos efectuados en la Clínica Primero de Mayo de los Seguros Sociales de Bogotá. Las historias consultadas corresponden a pacientes ingresadas en los dos últimos meses de 1952 y en los siete primeros meses de 1953. Los accidentes analizados son los mater nos únicamente.

En 3.800 partos se registraron 100 accidentes de distinta naturaleza, lo cual da un índice de $2,63 \%$.

Frecuencia de los Accidentes Maternos de Parto: $2.63 \%$.

Los principales accidentes estuvieron representandos por hemorragias del alumbramiento, del post-parto y de la operación cesárea; retención de la placenta o expulsión retardada de la misma; desgarros cervicales tanto de los partos intervenidos con forceps como de los partos espontáneos; desgarros profundos de la vagina y del periné; hemorragia y shock asociados.

En el cuadro $1^{0}$ se anota: clase de accidente, número de casos y un índice de frecuencia para cada tipo de accidente.

\section{CUADRO No 1}

\begin{tabular}{|l|c|c|}
\hline \multicolumn{1}{|c|}{ A C C I D E N T E } & No DE CASOS & $\begin{array}{c}\text { INDICE DE } \\
\text { FRECUENCIA }\end{array}$ \\
\hline Hemorragia & 51 & $13,4 \%^{\circ}$ \\
\hline Retención de la placenta & 17 & $4,4 \%^{\circ}$ \\
\hline Shock & 10 & $2,6 \%^{\circ}$ \\
\hline Desgarros cervica!s & 9 & $2,3 \%^{\circ}$ \\
\hline Desgarros vagino-perineales & 6 & $1,5 \%^{\circ}$ \\
\hline Hemorragia y shock & 4 & $1,05 \%^{\circ}$ \\
\hline Varios & 3 & $0,7 \%^{\circ}$ \\
\hline
\end{tabular}


Se han considerado únicamente los desgarros vagino-perineales extensos, sobre todo los que se han extendido ampliamente en los tejidos vaginales y que han constituído por su profundidad, extensión y hemorragia un verdadero accidente del parto. Varios, corresponde a un caso de tetanización uterina, uno de síncope anestésico y uno de ruptura de várice vulvar con abundante hemorragia.

Accidentes del parto en 100 casos, según el número de embarazos de las pacientes:

\section{CUAADRO No II}

\section{ACCIDENTES DEL PARTO EN LAS PRIMIPARAS}

\begin{tabular}{l|c|c|c|c|c|c|c|c|c|c}
\hline No. de embarazo & I & II & III & IV & V & VI & VII & VIII & IX & X \\
\cline { 2 - 6 } No. de casos & 37 & 33 & 10 & 7 & 5 & 2 & 1 & 1 & 2 & 2 \\
\hline
\end{tabular}

Son más frecuentes los accidentes en Para I y Para II, aparentemente, porque de las pacientes que ingresan a la Clínica, la mayoría pertenecen a a estos dos grupos ( ${ }^{1}$ ).

En los cuadros III, IV, V, VI y VII aparecen los accidentes sufridos por Para I, II, III, IV y V.

\section{CUADRO III}

\begin{tabular}{|l|c|c|}
\hline A C C I D E N T E S & No DE CASOS & Porcentaje \\
\hline $\begin{array}{l}\text { Hemoragia del alumbramiento y del } \\
\text { post-parto }\end{array}$ & 17 & $45,9 \%$ \\
\hline Shock obstétrico & 7 & $18,9 \%$ \\
\hline Grandes desgarros vagino-perineales & 4 & $10,9 \%$ \\
\hline Desgarros del cuello & 3 & $8,1 \%$ \\
\hline Retención de la placenta & 3 & $\mathbf{8 , 1 \%}$ \\
\hline Tetanización uterina. & 1 & $\mathbf{2 , 7 \%}$ \\
\hline Hemorragia en operación cesárea. & 1 & $2,7 \%$ \\
\hline Ruptura de la varice vulvar & 37 & $2,7 \%$ \\
\hline
\end{tabular}

Dos casos de hemorragia estuvieron ligados respectivamente a retención de lit placenta y a retención de las membranas ovulares. 


\section{CUIADRO IV}

ACCIDENTES DEL PARTO EN LAS SECUNDIPARAS

\begin{tabular}{|l|c|c|}
\hline \multicolumn{1}{|c|}{ A C C I DE N TES } & No DE CASOS & Porcentaje \\
\hline $\begin{array}{l}\text { Hemorragia del alumbramiento y del } \\
\text { post-parto }\end{array}$ & 24 & $72,73 \%$ \\
\hline Retención de la placenta & 5 & $15,15 \%$ \\
\hline Desgarros del cuello & $\mathbf{3}$ & $9,09 \%$ \\
\hline Shock obstétrico & 1 & $3,03 \%$ \\
\hline & TOTAL & $100 \%$ \\
\hline
\end{tabular}

Un caso de retención de la placenta estuvo ligado a shock obstétrico. Tres casos de shock estuvieron ligados a hemorragias; un gran desgarro vagino-perineal estuvo acompañado de hemorragia.

\section{CUADRO V}

ACCIDENTES DEL PARTO EN PARA III

\begin{tabular}{|l|c|c|}
\hline \multicolumn{1}{|c|}{ A C C I D EN TE s } & No DE CASOS & PORCENTAJE \\
\hline $\begin{array}{l}\text { Hemorragias del alumbramiento y del } \\
\text { post-parto }\end{array}$ & 6 & $60 \%$ \\
\hline $\begin{array}{l}\text { Retención de la placenta } \\
\text { Shock obstétrico }\end{array}$ & 3 & $30 \%$ \\
\hline & 1 & $10 \%$ \\
\hline
\end{tabular}

Dos casos de hemorragia estuvieron ligados respectivamente a apopleji: útero-placentaria y a un desgarro cervical intenso. 


\section{CUADRO VI}

ACCIDENTES DEL PARTO EN PARA IV

\begin{tabular}{|l|l|}
\hline & No DE CASOS \\
\hline $\begin{array}{c}\text { Hemorragias del alumbramiento y del } \\
\text { post-parto }\end{array}$ & 4 \\
\hline Desgarros cervicales & 1 \\
\hline Retención de la placenta & 1 \\
\hline Síncope anestésico & 1 \\
\hline & TOTAL \\
\hline
\end{tabular}

Dos casos de hemorragia aparecieron en el curso de operaciones cesáreas. sobre la abertura del segmento.

\section{CUADRO VII}

ACCIDENTES DEL PARTO EN PARA V

\begin{tabular}{|l|l|}
\hline $\begin{array}{l}\text { Aemorragia del alumbramiento y del } \\
\text { post-parto }\end{array}$ & No DE CAsos \\
\hline Desgarros cervicales & 2 \\
\hline Desgarros vagino-perineales extensos & 1 \\
\hline Retención de la placenta & 1 \\
\hline & TOTAL \\
\hline
\end{tabular}

El caso anotado de desgarro vagino-perinea! extenso estuvo complicado con la abertura de una fístula recto vaginal operada anteriormente. Tanto el desgarro como la fístula fueron tratados después del parto con buen resultado.

Accidentes del parto en Para V1: 2 casos: $\left\{\begin{array}{l}19 \text { Retención de la placenta. } \\ 20 \text { Shock obstétrico. }\end{array}\right.$ 
Accidentes del parto en Para VIII: I casos \& Retención de la placenta.

Accidentes del parto en Para 1X: 2 casos $\} \begin{aligned} & \text { 1" Tctanización uterina. } \\ & \text { 2" Hemorragia post-partum. }\end{aligned}$

Accidentes del parto en Para X: 2 casos

19. Hemorragia en op. césarea.

29 Retención de placenta, hemorragia y shock.

\section{PARTOS GEMELARES}

Dos casos (Historias números 27 y 60). Ambas Para 1I. La primera hipertensa: se hizo aplicación de forceps y versión y extracción podálica para el $1^{\circ}$ y $2^{\circ}$ feto sucesivamente; presentó retención de la placenta. Se practicó alumbramiento manual. La segunda tuvo el parto espontáneo. Presentó hemorragia post-partum. Se hizo taponamiento uterino.

\section{CUADRO VIII}

EVOLUCION DEL EMBARAZO EN LOS 100 CASOS

\begin{tabular}{|l|c|}
\hline \multicolumn{1}{|c|}{ TIPO DE EMBARAZO } & No DE CASOS \\
\hline Normal & 65 \\
\hline Sin dato & 28 \\
\hline Preeclampsia & 3 \\
\hline Pl. previa & 2 \\
\hline Hidramnios & 1 \\
\hline Hipertensión & 1 \\
\hline & TOTAL \\
\hline
\end{tabular}

Por defecto de estadística muchas historias aparecen $\sin$ datos.

Accidentes del parto en la precclampsia

Hemorragia del alembamiento: 2 casos.

Shoch, defuncion matema: I caso. 
Accidentes del parto en la placenta previa (dos casos tratados con cesárea): Hemorragia abundante sobre la abertura del segmento.

Accidentes del parto en el hidramnios: Hemorragia del alumbramiento.

Accidentes del parto en la Hipertensión Arterial: Historia No 27 citada en partos gemelares.

\section{CUADRO IX}

CONDUCTA Y DROGAS EN EL TRABAJO Y PRE-PARTO

\begin{tabular}{|l|c|}
\hline \multicolumn{1}{|c|}{ OBSERVAciones } & No DE CAsos \\
\hline Sin drogas o sin dato & 50 \\
\hline Ocitócicos (Pitocín) & 22 \\
\hline Sedantes y antiespasmódicos & 15 \\
\hline Mezcla de sedantes y ocitócicos & 9 \\
\hline Dilatación artificial del cuello & 4 \\
\hline & TOTAL \\
\hline
\end{tabular}

Los 50 casos, sin droga o sin datos, o ingresaron directamente de la calle a la sala de partos o se olvidó anotar en la historia los datos respectivos. Esta cifra, si no es exacta, sí es aproximada: la conducta de muchos durante el trabajo se reduce a la aplicación de un lavado intestinal, por principio, o porque las condiciones de la paciente no requieren otra medida. Estas pacientes sin medicación sufrieron diversos accidentes en el parto: hemorragias del alumbramiento y del post-parto; expulsión demorada de la placenta; shock; desgarros cervicales y grandes desgarros vagino-perineales.

El Pitocín se usó en forma prudente: 1, 2 y 5 unidades como máximo.

El caso de 10 u. (aplicadas a domicilio. Historia Nọ 3) presentó tetanización uterina.

Como sedantes y antiespasmódicos se uso el atrinal, los barbitúricos, el Demerol y el Trilene. En 9 casos estuvieron asociados al Pitocín.

Uno de los casos de dilatación artificial del cuello presentó un desgarro cervical y abundante hemorragia por esta causa. Los otros tres casos de dilatación del cuello fueron seguidos de aplicación de forceps. En uno de estos se presentó shock obstétrico.

\section{DUIRACION DEL TRABAJO Y ACCIDENTES DEL PARTO}

Estos accidentes aparecieron con los más diversos tiempos de duración del trabajo, desde los más cortos hasta los más prolongaden. Hubo extracciones del feto antes del trabajo. (Historia $V 0$ 21): $\left(\mathrm{I}_{1}\right.$ caso de apoplejía útero 
placentaria tratado con operación cesárea sin histerectomia que presentó hemorragia persistente en el post-parto. Hubo trabajos de l y 2 horas así como también de 48 y 52 horas. Los accidentes acaccidos en estos dos tipos de trabajo fueron más o menos parecidos: hemorragia, shock, desgarros cervicales y vagino-perineales extensos.

\section{CLIADRO X}

\section{ANESTESIA EMPLEADA}

\begin{tabular}{|c|c|c|c|c|c|c|c|}
\hline $\begin{array}{c}\text { Tricloro- } \\
\text { etileno }\end{array}$ & $\begin{array}{c}\text { Ciclo- } \\
\text { propano }\end{array}$ & Eter & $\begin{array}{c}\text { Trilene- } \\
\text { ciclo }\end{array}$ & $\begin{array}{c}\text { Trilene } \\
\text { procaina }\end{array}$ & Pentotal & $\begin{array}{c}\text { Pentotal } \\
\text { ciclo }\end{array}$ & $\begin{array}{c}\text { Sin } \\
\text { anestesia }\end{array}$ \\
\hline 41 & 38 & 4 & 4 & 3 & 1 & 1 & 8 \\
\hline
\end{tabular}

Es difícil juzgar con precisión qué agente anestésico es el responsable de un mayor número de accidentes del parto. Para esto se necesitaría tener el dato de los anestésicos empleados en los 3.800 casos consultados. En todo caso, con una atenta revisión del cuadro general final se echa de ver que el mayor número de accidentes por anestesia, le son imputables al tricloroetileno.

\section{CUADRO XI}

INTERVENCIONES PRACTICADAS

\begin{tabular}{|c|c|c|c|}
\hline FORCEPS & CESAREA & $\begin{array}{c}\text { DILAT. ARTIF.DEL } \\
\text { DEL CUELLO }\end{array}$ & $\begin{array}{c}\text { EXTR. POD } \\
\text { VERSION Y }\end{array}$ \\
\hline 41 & 6 & 4 & 1 \\
\hline
\end{tabular}

Los accidentes imputables al forceps consistieron en shock especialmente en una aplicación alta y en cuatro casos en que la anestesia fue el tricloroetileno aunque también hubo casos de shock con el ciclo.

Las complicaciones de la cesárea consisten en hemorragias abundantes sobre la abertura del segmento. En el caso de la cesárea conservadora por apoplejía útero-placentaria la hemorragia apareció en el post-operatorio inmediato. En todas se uso el ciclopropano.

En los casos de dilatación artificial del cucllo, uno presento desgarro cervical y otro shock obstétrico. Tres de los cuatro casos fueron seguidos de apli. caciones de forceps. 


\section{CUIADRO XII}

ACCIDENTES DEL PARTO

\begin{tabular}{|c|c|c|c|c|c|c|c|c|}
\hline \multirow{2}{*}{ ACCIDENTES } & \multirow{2}{*}{$\begin{array}{c}\text { No. de } \\
\text { casos }\end{array}$} & \multicolumn{2}{|c|}{ PARTOS INTERV. } & \multirow{2}{*}{$\begin{array}{l}\text { Partos } \\
\text { espónt. }\end{array}$} & \multicolumn{4}{|c|}{ A NESTESIA } \\
\hline & & Foorc. & $\overline{\text { Cesárea }}$ & & Trilene & ciclo & Varios & Sin \\
\hline Hemoiragia & 51 & 18 & 6 & 27 & 22 & 18 & 5 & 6 \\
\hline $\begin{array}{l}\text { Retención de la } \\
\text { pacenta }\end{array}$ & 17 & 3 & 0 & 14 & 10 & 4 & 2 & 1 \\
\hline Shock & 10 & 8 & 0 & 2 & 4 & 6 & 0 & 0 \\
\hline $\begin{array}{l}\text { Desgarros } \\
\text { cervicales }\end{array}$ & 9 & 5 & 0 & 4 & 2 & 4 & 3 & 0 \\
\hline $\begin{array}{l}\text { Desgarros } \\
\text { v. p }\end{array}$ & 6 & 4 & 0 & 2 & 0 & 4 & 2 & 0 \\
\hline $\begin{array}{l}\text { Hemorragia } \\
\text { y shock }\end{array}$ & 4 & 1 & 0 & 3 & 2 & 1 & 0 & 1 \\
\hline Varios & 3 & 2 & 0 & 1 & 1 & 1 & 1 & 0 \\
\hline TOTALES & 100 & 41 & 0 & 53 & 41 & 38 & 13 & 8 \\
\hline
\end{tabular}

Cuatro casos de retención placentaria presentaron hemorragia y shock. Una de las pacientes llegó a la Clínica después del nacimiento del niño por retención placentaria. Se le practicó extracción manual.

Un caso de desgarro vagino-perineal estuvo asociado a shock obstétrico. Varios comprende: un caso de tetanización uterina; uno de síncope anestésico y uno de ruptura de várice vulvar. ciclo.

Varios, en anestesia, corresponde a éter, pentotal, trilene-ciclo y pentotal-

\section{TRATAMIENTO DE LOS ACCIDENTES DEL PARTO}

Hay constancia en las historias clínica de las siguientes medidas:

Transfusiones de sangre o plasma .............. 27 casos.

Suturas diversas (periné, vagina, cuello, segmento) ...... 21

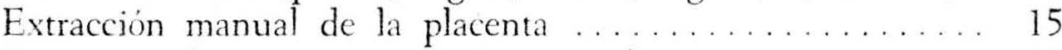

Ocitócicos (se presume mayor número) ........... 12

Taponamientos uterinos $\ldots \ldots \ldots \ldots \ldots \ldots \ldots \ldots \ldots \ldots$

Sucros dextrosados (se presume mayor número) ........ 5

Estas medidas estuvicron complementadas la mayoría de las veces con tónicos cardio-respiratorios, dexosicorticosterona y 02 . 


\section{RESLILTADOS}

Mortalidad materna: l caso (Historia No 9t) a consecuencia de un shock obstétrico en una preeclámptica, con feto grande, intervenida con aplicación de forceps.

Mortinatalidad: 4 casos (Historias númcros 21, 41, 79 y 94) por desprendimiento prematuro de la placenta; por tetanización uterina; un feto muerto y macerado; y por preeclampsia en un parto laborioso.

\section{COMENTARIOS}

Es importante el estudio de los accidentes del parto por cuanto consitituye un método preventivo de la morbilidad y aún de la mortalidad maternas.

Hemorragia. - Las hemorragias estudiadas, las más frecuentes además, corresponden a las del alumbramiento y a las del post-parto. En su etiología pode mos considerar: causas dependientes del organismo materno y causas no dependientes de él.

10-Como causas dependientes del organismo materno citaremos, como más frecuentes: atonía uterina, retención parcial o total de la placenta y diátesis hemorrágica $\left({ }^{2}\right)$.

$2^{\circ}$ - Como causas no dependientes de la madre citaremos fallas y errores en la conducción del parto y del alumbramiento, tales como el uso desmedido de sedantes y anestésicos, morfina, barbitúricos, tricloro etileno $\left({ }^{3}\right)$; presiones violentas ejercidas sobre el fondo uterino durante el desprendimiento y aún durante el descenso de la presentación; maniobras intempestivas ejercidas sobre la matriz o sobre el cordón umbilical durante el periodo del alumbramiento. En cuanto al tratamiento, son de suma importancia las medidas preventivas consistentes en la observación, de una conducta racional durante el parto y el alumbramiento, reduciendo las intervenciones y el suministro de drogas a lo estrictamente necesario.

Algunos usan, preventivamente, la Ergonovina o el Pitocín aplicados por vía venosa en el momento del desprendimiento de la presentación, o bien el Pitocín intravenoso, aplicado poco después de la expulsión del feto con lo cual consigue la expulsión ma pronto, de la placenta, prevenir la hemorragia o whibirla, si la hay.

En caso de pacientes con antecedente de profusas hemorragias del alum. bramiento en partos anteriores es conveniente estar preparados, con transfusion. acitocicos, equipo para revisión uterina, curctaje y taponamiento. Procediendo 
en forma metódica con habilidad, prontitud y contando con estos medios es raro que el accidente llegue a ser fatal.

Ante este tipo de hemorragia, nos hemos trazado esta línea de conducta y es la que personalmente seguimos cuando el caso se presenta:

19-Si la placenta no ha sido expulsada, practicamos la inducción del alumbramiento, con 2 ó 3 u. de Pitocín i.v. En esta forma, se logra generalmente, expulsar la placenta y detener la hemorragia. Si no se consiguen estos fines consideramos que la placenta está parcial o totalmente desprendida pero retenida en la cavidad uterina por un anillo de constricción. Aplicamos entonces anestesia general a la paciente con lo cual se consigue desvanecer el anillo de constricción y restablecer la sinergia del músculo uterino; así la placenta sale por los medios naturales o ayudada con expresión del cuerpo de la matriz.

Aclaramos en todo caso, que si la hemorragia se manifiesta abundante desde el principio, procedemos sin dilación ninguna a efectuar la extracción manual, seguida de revisión digital y visual, del tractus genital, escobillonaje con mertiolate, taponamiento y ocitócicos según las condiciones del caso.

20-Si ya se expulsó la placenta y la hemorragia persiste o se produce, procedemos según la intensidad de la misma. Si la hemorragia es escasa aplicamos Ergonovina o Pitocín (2-3 u.) por vía endovenosa ya sea solo o disuelto en 500 c.c. de dextrosa al 5\%. Algunos recomiendan la aplicación de varias dosis de Pitocín y Ergotrato, alternados, con media hora de intervalo ( ${ }^{4}$ ). Si la hemorrgia es abundante hacemos revisión del tractus genital, con mayor razón si ha habido intervenciones (forceps, basiotribo, versión, etc.). Procediendo con rapidez, extraemos restos ovulares si los hay y practicamos en seguida un taponamiento apretado, no con mecha, sino con varias compresas embebidas en mertiolate y anudadas unas a continuación de otras. El taponamiento, correctamente hecho, es eficaz en una gran mayoría de los casos y practicado en con diciones asépticas y con ayuda de antibióticos, es inocuo para las pacientes.

Salvo el fracaso de estas medidas, descartamos el recurso de la histerectomía. Consideramos esta medida, como una eventualidad rara, y de difícil elección el momento oportuno para practicarla: si la paciente está en relativas buenas condiciones es posible que la hemorragia ceda con medios no quirúrgicos aplicados correctamente; si la paciente está en malas condiciones es posible que no soporte la intervención y no sobreviva. Anteriormente los autores indicaban con más frecuencia esta intervención debido a la carencia de recursos que, como la san gre, y el plasma, nos son hoy día de fácil y pronta adquisicion gracias a las cuales logramos ahora sostener las condiciones de la paciente mientras practicamos suturas de tejidos desgarrados, revisiones, raspados y taponamientos.

Hemoragias en la operacion cexirea. - Estas hemorragias se presentan a veces en la disociación del segmento pero son evitables en todo caso disponiendo 
de habilidad quirúrgica y técnica apropiada. Casi no se presentan usando la raquianestesia debido a que con ésta se aumenta el tono uterino y es mayor la retractilidad al expulsarse el feto. Esto sucede en menor grado con la anestesia general, de ahí que usemos y recomendemos la raquianestesia en la cesárea, siempre que lo permita el estado de la madre.

Aparece también en pacientes con degeneración fibrosa de los tejidos, como acontece en algunas multiparas y en primíparas de edad avanzada. Estas mismas pacientes suelen presentar grandes senos venosos sobre la cara anterior del segmento. Es muy conveniente ligarlos y seccionarlos antes de efectuar la disociación del segmento para evitar su ruptura con pro ducción de abundantísima hemorragia que puede hacer peligrar la vida de la madre.

En caso de cesáreas iterativas, con existencia de cicatriz fibrosa sobre el segmento, la disociación no se hace con las debidas precauciones es posible producir desgarros irregulares que se extiendan hasta el cuerpo de la matriz y hasta los fondos de saco vaginales con producción de abundante hemorragia difícil de detener. En estos casos acostumbramos seccionar transversalmente el segmento sobre la cicatriz fibrosa, con tijera o bisturi sobre una sonda acanalada. Desechamos las maneras bruscas y precipitadas tanto en la abertura del segmento como en la extracción de la cabeza fetal, responsables en muchos casos de estos graves accidentes maternos.

Hemorragia en la apoplejía útero-placentaria. - Opinan algunos que en esta entidad debe hacerse siempre histerectomía aún en casos de lesión leve del músculo uterino por el peligro de la hemorragia incoercible subsecuente. Nosotros procedemos en ésta forma: en caso de gran degeneración de la matriz, histerectomía. En casos leves si la matriz se contrae y responde a los ocitócicos: adoptamos una conducta conservadora. Hace pensar bastante el histerectomizar a una mujer tanto más si es joven, primípara y si ha perdido su primer hijo. Al seguir esta norma debemos estar prevenidos con transfusión y equipo quirúrgico listo para intervenir si la hemorragia aparece.

En una paciente de la Caja del Municipio, operada el año pasado en la Clínica Magdalena, con lesión leve de la matriz, la hemorragia apareció abundante al estar suturando el peritoneo segmentario después de la cesárea. Practicamos en seguida la histerectomía; la paciente sobrevivió gracias al abundante suministro de angre. En el caso citado en este trabajo la hemorragia se hizo presente poco después de la cesárea; fue detenida y se evitó la histerectomía gracias a un taponamiento intrauterino con Oxiciel y con gasa.

Cuando sea necesario practicar una transtusion es imprescindible la deter minación del factor Rh a fin de asegurar que la sangre sea compatible y evitar los graves inconvenientes de la inmunización anti-Rh $\left(^{5}\right)$. 
Retención de la placenta. - Es el accidente más frecuente (4,4\%) después de las hemorragias del alumbramiento y del post-partum. Los casos analizados en este capítulo posiblemente se refieran a expulsiones más o menos de moradas de la placenta, pues ya es raro que un obstetra espere por espacio de una, dos o más horas que se cumpla este último periodo del parto, según lo propuesto por los clásicos, debido a los peligros próximos o remotos que esta medida entraña hemorragia, shock, infección, intranquilidad y desconfianza en el ánimo de la paciente. Nuestra conducta en estos casos depende de si hay o no hemorragia. Si la hay procedemos según lo anotado en el capítulo referente a hemorragias. Si no la hay, procuramos en un principio la inducción del alumbramiento con Pitocín intravenoso. Si fracasa, consideramos la posibilidad de modi ficaciones anatómicas y funcionales del músculo uterino que se oponen a la realización del alumbramiento: degeneración fibrosa de la matriz, miomas, atonía irreductible, anillos de constricción, adherencia anormal de la placenta, placenta acreta, etc. Dada esta posibilidad sería inútil una espectativa prolongada. Por tanto acudimos luégo a practicar la extracción manual.

Nuestra impresión acerca de este método, según experiencia nuestra, es de que practicado con técnica apropiada, en condiciones de asepsia y complementado con antibióticos, no es perjudicial para la paciente y no implica morbilidad alguna. Nos parece que es más conveniente hacer la extracción manual pronto que después de una o dos horas, pues para ese tiempo las condiciones maternas pueden ser ya desfavorables por anemia y descenso de las defensas orgánicas, puesto que se ha llegado a demostrar que las pacientes anémicas son más susceptibles a la infección y que los antibióticos poseen una acción más efectiva en pacientes no anémicas $\left({ }^{6}\right)$.

Recalcamos que la profilaxis de la retención de los anexos ovulares consiste en no entorpecer el mecanismo del alumbramiento fisiológico y en evitar maniobras intempestivas como expresiones fuertes sobre el fondo uterino y tracciones sobre el cordón umbilical $\left({ }^{i}\right)$.

Shock obstétrico. - Según nuestra estadística este accidente se presenta en el 2,6\% de los partos. En cuanto al diagnóstico nos valemos de los signos puramente clínicos; nuestro medio no está familiarizado aún con algunos datos de laboratorio como hemoconcentración y aumento del potasio plasmático, que gozan de la aceptación en el diagnóstico y pronós ico del shock por parte de autores americanos y argentinos ("). Sobre su profilaxis anotamos los siguientepuntos: control prenatal adecuado, precención de ha gestosis, buena te nica qui rúrgica, y operaciones breves; sospercharlo y estar prevenidos en caso de trabajos prolongados en pacientes obesas, gestósicas o de constitucion neuro-cndocrina inestable (Pérez N. L. op. cit.), en partos laborioses sobre todo con difíciles aplicaciones de forcep basiotribo, versiones y extracciones podalicas. Nunca con denaremos to suficiente las ceppresiones grosicras, a to Kristeller, responsables en 
muchos casos no sólo de shock, sino de hemorragias y retención de los anexos ovulares.

En cuanto al tratamiento, además de las medidas básicas usuales, plasma, (02, tónicos circulatorios, corteza suprarrenal, etc., creemos que se debe hacer un estudio serio sobre la acción benéfica del ACTH. En un caso personal obtuvimos magnifico y espectacular resultado. Tenemos noticia también de buenos cfectos en algunos pocos casos de los colegas tanto del ICCS. como de clínicas particulares.

Desgarros cervicales y vagino-perineales. - Respecto de los desgarros cervicales hacemos notar su ocurrencia tanto en partos espontáneos como en partos intervenidos y ocasionados por la expulsión rápida del feto antes de la dilatación completa, por aplicaciones de forceps, de basiotribo y por dilataciones artificiales. A ello puede contribuír también un estado anatómico especial del cuello como fibrosis, edema o infección. Es conveniente después de efectuar las intervenciones arriba citadas, verificar la integridad del tractus genital, tanto manual como visualmente, ayudados de instrumental apropiado como valvas y pinzas cervicales, para localizar y poder reparar en debida forma las lesiones acaecidas.

Los desgarros vagino-perineales estudiados aquí no se refieren a los de $1^{\circ}$, $2^{\circ}$ y $3^{\circ}$ grados. Consideramos que es poca la frecuencia de estos y pequeña su importancia ya que la práctica de la episiotomía es observada por la mayoría de los obstetras, con lo cual ellos son evitados. Las lesiones consideradas aquí se refieren a los grandes destrozos de la vagina que se extienden a veces hasta la fosa isquiorectal, los fondos de saco y la base de los ligamentos anchos y que por su profundidad, extensión y hemorragia constituyen un serio accidente del parto. En su producción concontribuyen factores maternos como estrechez y reducción vulvo vaginal, infantilismo genital, esclerosis vaginal y colpitis agudas o crónicas. Son determinados por la expulsion brusca del polo fetal ya en el parto espontáneo o en el intervenido con Kristeller o con forceps. Su aparición es inevitable cuando el obstetra precipita la expulsión del feto en una u otra forma antes de que la resistencia de los tejidos vagino-perineales haya sido vencida en forma natural y progresiva.

Es conveniente pues que el intervencionismo de este período del parto sea mesurado y prudente. En caso de estrechez vagino-perineal muy acentuada se observará un conducta de espectativa y llegado el momento se practicará episiotomía bilateral amplia. Son raros los casos de infantilismo genital en que se leba acudir a la vía alta debido al obstáculo presentado por las partes blandas.

Anestesia. - En las historias consultadas encontramos tan sólo un caso de sincope anestésico producido por el ciclopropano (Historia $N^{0} 45$ ). Por expe riencia personal y por to que hemos observado, nos parece, que los accidentes por anestesia en los partos son más frecuentes sobre todo con el trilene. Sucede 
que el obstetra olvida la mayor parte de las veces dejar consignados estos percances anestésicos en la historia clínica. Personalmente somos muy poco adictos al trilenc; con él hemos observado en la madre, taquipneas, bradipneas, aritmias y cianosis; shock, retención de la placenta y hemorragias del alumbramiento y del post-parto. Estos efectos pueden depender de la droga misma o ser debidos a una mala técnica de la administración como cuando es aplicado por aficionados, enfermeras, o internos novatos y por anestesiólogos. Esta droga debe ser aplicada bajo vigilancia médica estricta; no se debe usar en gestósicas, hepáticas ni cardiorrenales $\left({ }^{3}\right)$. Lo usamos forzados por las circunstancias, por no haber otra anestesia en el ICCS generalmente. En caso de aplicarlo, lo hacemos en dosis medianas para el desprendimiento en partos espontáneos complementado con anestesia local cuando hay necesidad de practicar episiotomía.

Rechazamos el trilene para intervenciones que necesitan anestesia profunda: dilataciones artificiales del cuello, curetaje, forceps, basiotribo, versión interna, etc.

Debe tenerse en cuenta que el trilene se debe usar como analgesia y no como anestesia. Pérez M. L. le anota como inconveniente el producir una marcada atonía uterina; reduce la contractilidad uterina en el $31 \%$ de las pacientes; de ahí la producción de hemorragias del alumbramiento y del post-parto aunque él no las ha observado debido a la medicación ocitócica profiláctica aplicada tan pronto termina el parto ("). Ferreira le anota iguales inconvenientes, tanto que para prevenir las hemorragias del período placentario y del post-parto, usa sistemáticamente una inyección de Methergin intravenoso en el momento de la salida del hombro anterior del feto o inmediatamente después de su total expulsión. (op. cit. loc. cit.) Recalcamos, finalmente que la analgesia y la anestesia obstétricas deben estar encomendadas a manos expertas.

\section{CONCLUSIONES}

1? - Los accidentes maternos de] parto se presentan con una frecuencia del $2.6 \%$.

29-Los principales accidentes están constituídos por hemorragias del alumbramiento y del post-parto $(13.4 \% 0)$; retención de los anexos ovulares $(4.4 \% 0)$. shock obstétrico $(2.6 \% 0)$; desgarros del cuello uterino sobre todo en partos intervenidos $(2.3 \% 0)$; desgarros vagine-perineales profundo ( $1.5 \% 0)$; shock y hemorragia combinados $(1 \% 0)$.

30- Las pacientes en quienes más se presentaron accidentes fueron en las de primero, segundo y tercer parto. Aclaramos que las paciente de este tipo son las que en mayor numero son atendidas en la Clínica Primero de Mayo. 
4ํ-El accidente más frecuente en las primíparas, sccundíparas y tercíparas, fue la hemorragia del alumbramiento y del post-alumbramiento. También en las primíparas fue notoria la frecuencia de los desgarros vagino-perineales profundos.

50.-En la producción de estos accidentes influyen, además de factores mater nos, fallas técnicas en la conducción del parto y del alumbramiento.

60- Los datos referentes a la relación entre el accidente del parto y ciertos aspec. tos del embarazo y del paro (gestosis, drogas aplicadas durante el trabajo) son apenas aproximadas, debido a insuficiencia de las historias y a defectos del sistema estadístico

79-La mejor prevención de los accidentes del parto consiste en tratar de perturbar lo menos posible su fisiologismo natural.

8--Parece que el ACTH sirve en el shock obstétrico. Convendría un estudio más extenso sobre este punto.

90- Se debe tener más en cuenta, los métodos de analgesia y anestesia obstétricas y deben ser manejados por manos expertas.

\section{B I B L I O G R A F I A}

1.-Carvajal-Barrera: "Bioestadigrafía y Análisis clínico comparado de 20.000 casos obstétricos. Rev. Col. de Ob. y Gin. Vol. V. 82; 1953.

2.-Stoeckel: "Tratado de obstetricia". Ed. III, 1945. Pág. 535.

3.-Ferreira: "Analgesia obstétrica con tricloroetileno". Rev. Española de Obst. y Gin. Tomo XII, No 68. Págs. 101, 102, 103.

4.-Murphy, Roselle: "The management of the third stage of Labor employin Pitocin and Methergine". Am. J. Obst. Gynec. Vol. 64, No 2. Pag. 419; 1952.

5.-Plata-Rueda: "Conducta en la gestante Rh. negativa". Inédito. Primera Conv. Nal. de Obst. y Gin. 1953. Bogotá.

6.-Collins, Smith, Burman: "Sangre y vasos onguíneos en Obst. y Gin.". Citado por Rev. Col. de Obst. y Gin. Vol. V: 115; 1953.

7.-Pérez Manuel Luis: "Tratado de Obstetricia". Ed. V, Vol. 2, Pág. 1.005.

8.-Moragues-Bernat: "Clínica obstétrica". Ed. III, Pág. 728.

9. - Pérez M. L.: "Experiencia con el Trilene en la práctica privada". Obst. Ginec. Lat. Amer. Vol. XI. No 1-2, Pág. 33; 1953. 